\title{
The intriguing association between patent foramen ovale and atrial fibrillation
}

\author{
J David Spence ${ }^{1-3}$, Luciano A Sposato ${ }^{2-7}$ \\ 'Stroke Prevention \& Atherosclerosis Research Centre, Robarts Research Institute, Western University, London, Ontario, Canada \\ 2Department of Clinical Neurological Sciences, Western University, London, Ontario, Canada \\ ${ }^{3}$ Robarts Research Institute, Western University London, Ontario, Canada \\ ${ }^{4}$ Heart \& Brain Laboratory, Western University, London, Ontario, Canada \\ ${ }^{5}$ Department of Epidemiology and Biostatistics, Western University, London, Ontario, Canada \\ ${ }^{6}$ Department of Anatomy and Cell Biology, Western University, London, Ontario, Canada \\ ZLawson Health Research Institute, London, Ontario, Canada
}

\section{Related article}

by Kiełbasa et al., see p. 756

\section{Correspondence to: J David Spence, CM, MD, FRCPC, FAHA \\ Stroke Prevention \& \\ Atherosclerosis Research \\ Centre, \\ Robarts Research Institute, \\ Western University, \\ 1400 Western Road, \\ London, Ontario, Canada \\ N6G 2V4, \\ phone: +15 199315731 , \\ e-mail: \\ dspence@robarts.ca \\ Copyright by the Author(s), 2021 \\ Kardiol Pol. 2021. \\ 79 (7-8): 725-727; \\ DOl: 10.33963/KP.a2021.0028 \\ Received: \\ May 30, 2021 \\ Revision accepted: \\ May 30, 2021 \\ Published online: \\ June 1, 2021}

In this issue of Kardiologia Polska, Kiełbasa et al. [1] evaluated the risk of atrial fibrillation (AF) recurrence in a cohort of 417 consecutive patients with paroxysmal AF undergoing cryoballoon ablation for pulmonary vein isolation (PVI). The left atrium was accessed transseptally in all cases; median follow-up time was 2 years (maximum 7 years). Recurrent AF was ascertained by 24-72-hour Holter monitoring at 3 and 6-9 months after ablation. Further electrocardiographic monitoring was advised yearly or in case of palpitations. Stepwise multivariable Cox regression analysis identified variables independently associated with the risk of recurrent AF. Overall, AF recurrence was documented in $25.7 \%$ of the study cohort after 11056 patient months of follow-up. Left atrial diameter $>40 \mathrm{~mm}$ (adjusted hazard ratio [aHR] 1.88, 95\% $\mathrm{Cl}, 1.23-2.87 ; P=0.004)$, low left atrial appendage flow velocity defined as $<45 \mathrm{~cm} / \mathrm{s}$ (aHR 1.63 , $95 \% \mathrm{Cl}, 1.06-2.49 ; P=0.02)$, and the presence of a patent foramen ovale (PFO) (aHR 1.79, 95\% Cl, $1.02-3.15 ; P=0.04$ ) were independently associated with the risk of AF recurrence. This study has several strengths, including the fairly large size, and adjustment of the multivariable models for all clinically relevant variables. The authors correctly acknowledged most of its limitations, including that it is a retrospective study based on data from a single center. The dichotomization of left atrial velocity may explain its signifi- cant association with $\mathrm{AF}$ recurrence. The authors used a cutoff derived from the same cohort in which they subsequently ran the multivariable regression analyses, so a significant association is not surprising.

The association between increased left atrial size and AF recurrence post ablation has been well-documented in previous studies [2]. In line with the findings of the study by Kiełbasa et al. [1], reduced left atrial appendage velocity has been found to be associated with increased risk of AF after cardiac surgery [3]. In our view, the most intriguing finding of this study is the $79 \%$ increased risk of AF recurrence associated with the presence of a PFO. This finding adds to consideration of important issues about the association between AF and PFO that we discuss below: (a) the high prevalence of concurrent $A F$ and PFO, and (b) the elevated risk of incident AF post PFO closure.

At least two studies have documented that catheter probing performed during pulmonary vein isolation procedures has a 2-3-fold higher PFO detection yield than transesophageal echocardiography (TEE) [4-6]. We hypothesized in 2020 that AF may be more common in patients with PFO [6]. In a study of the Risk of Paradoxical Embolism (ROPE) score, 40\% of patients with ESUS had a PFO, and AF was observed in $20.5 \%$ of PFO patients with a ROPE score of $0-6$, with a $53 \%$ risk of recurrent stroke 
over 10 years [7]. This association supports the suggestion that some patients with PFO might be treated better with anticoagulation than by closure of the PFO [8].

Since a PFO is present in $~ 25 \%$ of the population [9], it is surprising that Kielbasa et al. [1] found a PFO in only $12.7 \%$ of their patients, relying on TEE for diagnosis. Daher et al. [5] reported that among AF patients referred for PVI, 57\% had a PFO identified by catheter probing, but only $18.7 \%$ of patients had a PFO diagnosed by TEE. Small PFOs are less likely to be identified with echocardiography. Transcranial Doppler saline studies are more sensitive than echocardiography [10], perhaps in part because the adequacy of the Valsalva maneuver can be verified by the drop in blood velocity in the middle cerebral artery. It is possible that the pressure exerted on the interatrial septum with the ablation catheter may open otherwise collapsed PFOs.

The embolic risk of PFOs that are visualized only by catheter probing is likely low, although it has never been investigated. Alternatively, it is also possible that the PFO itself generates some degree of atrial vulnerability [11], creating the necessary physiological environment for the occurrence of $A F$, as mentioned by Kiełbasa et al. [1].

It can be hypothesized that individuals undergoing PFO closure have a specific susceptibility to develop cardiac arrhythmias triggered by the periprocedural manipulation of the left atrium. Left atrial trauma could result in local inflammation, a recognized trigger for AF [12]. Similarly, changes in left atrial intracavitary pressures and sudden distortions of its tridimensional architecture caused by the occlusion of the PFO could initiate AF. Although these mechanisms are plausible, they are likely influenced by other factors. First, as suggested by the frequent coexistence of paroxysmal AF and PFO in the study of Kiełbasa et al. [1], AF may constitute a latent subclinical condition in patients with PFO that becomes more active and clinically evident after the PFO is closed. In this hypothetical scenario, a PFO closure may lower the threshold for the occurrence of $A F$ paroxysms in patients with otherwise relatively inactive and subclinical AF. Additional possible factors explaining the elevated risk of paroxysmal AF after PFO closure are age and the presence of vascular risk factors or cardiovascular comorbidities. If the procedure itself was the only cause of $A F$ in patients with $\mathrm{PFO}$, the associated risk of $\mathrm{AF}$ relative to medical treatment alone would be expected to be similar in randomized clinical trials of PFO closure for secondary stroke prevention and clinical improvement of migraine with aura. However, in clinical trials of PFO closure for secondary stroke prevention, the risk of incident post-procedural AF was $5 \mathrm{x}$ higher compared to medical treatment [13], whereas in clinical trials of migraine prevention, there were no differences in the risk of incident AF between PFO closure and medical treatment [14]. Thus, discrepancies in the risk of Incident AF post PFO closure between patients with stroke and migraine are possibly explained by a higher age and a greater burden of risk factors and vascular co- morbidities in stroke patients. This is consistent with the observation by Strambo et al. [7] that among PFO patients with a ROPE score of $7-10$, only $3.1 \%$ had new-onset $A F$, and only $1.7 \%$ had stroke recurrence over 10 years.

The study by Kiełbasa et al. [1] provides additional evidence on the intriguing association between PFO and AF with a novel perspective that requires further testing in independent cohorts.

\section{Article information}

Acknowledgments: There was no funding for this work. Neither author has a conflict of interest related to this topic.

Conflict of interest: None declared.

Open access: This article is available in open access under Creative Common Attribution-Non-Commercial-No Derivatives 4.0 International (CC BY-NC-ND 4.0) license, allowing to download articles and share them with others as long as they credit the authors and the publisher, but without permission to change them in any way or use them commercially. For commercial use, please contact the journal office at kardiologiapolska@ptkardio.pl.

How to cite: Spence JD, Sposato LA. The intriguing association between patent foramen ovale and atrial fibrillation. Kardiol Pol. 2021; 79(7-8): 725-727, doi: 10.33963/KP.a2021.0028.

\section{REFERENCES}

1. Kiełbasa G, Bednarek A, Bednarski A, et al. Patent foramen ovale and left atrial appendage flow velocity predict atrial fibrillation recurrence post cryoballoon ablation. Kardiol Pol. 2021; 79(7-8): 756-764, doi: 10.33963/KP.a2021.0004, indexed in Pubmed: 34002841.

2. Jin $\mathrm{X}, \mathrm{Pan} \mathrm{J}, \mathrm{Wu} \mathrm{H}$, et al. Are left ventricular ejection fraction and left atrial diameter related to atrial fibrillation recurrence after catheter ablation?: a meta-analysis. Medicine (Baltimore). 2018; 97(20): e10822, doi: 10.1097/MD.0000000000010822, indexed in Pubmed: 29768386.

3. Ngai J, Leonard J, Echevarria G, et al. Left atrial appendage velocity as a predictor of atrial fibrillation after cardiac surgery. J Cardiothorac Vasc Anesth. 2016; 30(2): 413-417, doi: 10.1053/j.jvca.2015.08.023, indexed in Pubmed: 26706710.

4. Knecht S, Wright M, Lellouche N, et al. Impact of a patent foramen ovale on paroxysmal atrial fibrillation ablation. J Cardiovasc Electrophysiol. 2008; 19(12): 1236-1241, doi: 10.1111/j.1540-8167.2008.01260.x, indexed in Pubmed: 18662179.

5. Daher G, Hassanieh I, Malhotra N, et al. Patent foramen ovale prevalence in atrial fibrillation patients and its clinical significance; a single center experience. Int J Cardiol. 2020; 300: 165-167, doi: 10.1016/j.jjcard.2019.11.088, indexed in Pubmed: 31761403.

6. Sposato LA, Spence JD. Patent foramen ovale in patients with atrial fibrillation. Int J Cardiol. 2020; 300: 168-169, doi: 10.1016/j.ijcard.2019.11.125, indexed in Pubmed: 31785956.

7. Strambo D, Sirimarco G, Nannoni S, et al. Embolic stroke of undetermined source and patent foramen ovale: risk of paradoxical embolism score validation and atrial fibrillation prediction. Stroke. 2021; 52(5): 1643-1652, doi: 10.1161/STROKEAHA.120.032453, indexed in Pubmed: 33784832.

8. Spence JD. Anticoagulation in patients with embolic stroke of unknown source. Int J Stroke. 2019; 14(4):334-336, doi: 10.1177/1747493019826363, indexed in Pubmed: 30644794.

9. Hagen PT, Scholz DG, Edwards WD. Incidence and size of patent foramen ovale during the first 10 decades of life: an autopsy study of 965 normal hearts. Mayo Clin Proc. 1984; 59(1): 17-20, doi: 10.1016/s00256196(12)60336-x, indexed in Pubmed: 6694427.

10. Tobe J, Bogiatzi C, Munoz C, et al. Transcranial doppler is complementary to echocardiography for detection and risk stratification of patent foramen ovale. Can J Cardiol. 2016; 32(8): 986.e9-986.e16, doi: 10.1016/j. cjca.2015.12.009, indexed in Pubmed: 26952158.

11. Berthet $K$, Lavergne $T$, Cohen $A$, et al. Significant association of atrial vulnerability with atrial septal abnormalities in young patients with ischemic stroke of unknown cause. Stroke. 2000; 31(2): 398-403, doi: 10.1161/01. str.31.2.398, indexed in Pubmed: 10657412. 
12. Packer M. Characterization, pathogenesis, and clinical implications of inflammation-related atrial myopathy as an important cause of atrial fibrillation.J Am Heart Assoc. 2020; 9(7): e015343, doi: 10.1161/JAHA.119.015343, indexed in Pubmed: 32242478.

13. Chen JZJ, Thijs VN. Atrial fibrillation following patent foramen ovale closure: systematic review and meta-analysis of observational studies and clinical trials. Stroke. 2021; 52(5): 1653-1661, doi: 10.1161/STROKEAHA.120.030293, indexed in Pubmed: 33611943.

14. Kheiri B, Abdalla A, Osman M, et al. Percutaneous closure of patent foramen ovale in migraine: a meta-analysis of randomized clinical trials. JACC Cardiovasc Interv. 2018; 11(8): 816-818, doi: 10.1016/j.jcin.2018.01.232, indexed in Pubmed: 29673517. 